University of Nebraska - Lincoln

DigitalCommons@University of Nebraska - Lincoln

June 1987

\title{
Antiphase domain boundaries in the superconducting phase of the Y-Ba-Cu-O system
}

C.H. Chen

AT\&T Bell Laboratories, Murray Hill, New Jersey

J. Werder

AT\&T Bell Laboratories, Murray Hill, New Jersey

Sy_Hwang Liou

University of Nebraska-Lincoln, sliou@unl.edu

M. Hong

AT\&T Bell Laboratories, Murray Hill, New Jersey

Follow this and additional works at: https://digitalcommons.unl.edu/physicsliou

Part of the Physics Commons

Chen, C.H.; Werder, J.; Liou, Sy_Hwang; and Hong, M., "Antiphase domain boundaries in the superconducting phase of the Y-Ba-Cu-O system" (1987). Si-Hwang Liou Publications. 15. https://digitalcommons.unl.edu/physicsliou/15

This Article is brought to you for free and open access by the Research Papers in Physics and Astronomy at DigitalCommons@University of Nebraska - Lincoln. It has been accepted for inclusion in Si-Hwang Liou Publications by an authorized administrator of DigitalCommons@University of Nebraska - Lincoln. 


\title{
Antiphase domain boundaries in the superconducting phase of the $\mathrm{Y}-\mathrm{Ba}-\mathrm{Cu}-\mathrm{O}$ system
}

\author{
C. H. Chen, D. J. Werder, S. H. Liou, J. R. Kwo, and M. Hong \\ AT\&T Bell Laboratories, Murray Hill, New Jersey 07974-2070
}

(Received 23 March 1987)

\begin{abstract}
Transmission-electron-microscopy examination of the superconducting phase of the $\mathrm{Y}-\mathrm{Ba}-\mathrm{Cu}-\mathrm{O}$ system reveals the presence of dense antiphase boundaries. The structure of the superconducting phase was determined to be orthorhombic with space group $\operatorname{Pm} 2 m$. The orthorhombic $a$ and $b$ axes are found to alternate across the antiphase boundary which runs parallel to the [110] direction.
\end{abstract}

Since the report of possible high- $T_{c}$ superconductivity in the $\mathrm{La}-\mathrm{Ba}-\mathrm{Cu}-\mathrm{O}$ system ${ }^{1}$ and subsequent confirmation of the bulk superconductivity in this system, ${ }^{2-5}$ the search for even-higher- $T_{c}$ superconductors has been intensified in recent months. A recent report of superconductivity at 90-95 $\mathrm{K}$ in the $\mathrm{Y}-\mathrm{Ba}-\mathrm{Cu}-\mathrm{O}$ system ${ }^{6}$ has generated even greater excitement in the scientific community. The reported high- $T_{c}$ superconducting $\mathrm{Y}-\mathrm{Ba}-\mathrm{Cu}-\mathrm{O}$ system with optimal composition $\mathrm{Y}_{1.2} \mathrm{Ba}_{0.8} \mathrm{CuO}_{4-\delta}$ was found to be polycrystalline with a mixture of several different phases. Cava et al. ${ }^{7}$ further identified the superconducting phase as $\mathrm{Ba}_{2} \mathrm{YCu}_{3} \mathrm{O}_{9-\delta}$ from a single-phase sample. The structure of the superconducting phase has also been determined from powder $\mathrm{x}$-ray data ${ }^{7}$ as orthorhombic with a unit cell of $a=3.822 \AA, b=3.891 \AA$, and $c=11.677 \AA$.

In this Rapid Communication we present microstructure studies of the superconducting $\mathrm{Y}-\mathrm{Ba}-\mathrm{Cu}-\mathrm{O}$ system by transmission electron microscopy (TEM). We find that the superconducting phase is characterized by the presence of antiphase domain boundaries. Electron diffraction studies of the superconducting phase also yield an orthorhombic crystal structure with lattice parameters in good agreement with the $\mathrm{x}$-ray results. Furthermore, we determine the space group of the superconducting phase as $P m 2 m$.

The $\mathrm{Y}-\mathrm{Ba}-\mathrm{Cu}-\mathrm{O}$ compounds were prepared from $\mathrm{Y}_{2} \mathrm{O}_{3}$, $\mathrm{BaCO}_{3}$, and $\mathrm{CuO}$ powders pressed into pellets. The synthesis of such compounds of varying composition ratios will be reported elsewhere. ${ }^{8}$ In this work two bulk specimens were made, one with a composition of $\mathrm{Y}_{1.2} \mathrm{Ba}_{0.8} \mathrm{CuO}_{4-\delta}$ (sample 1), the other of $\mathrm{YBa}_{2} \mathrm{Cu}_{3} \mathrm{O}_{9-\delta}$ (sample 2). The pressed pellets were repeatedly ground and sintered at $950^{\circ} \mathrm{C}$ in air or $\mathrm{O}_{2}$ for $10 \mathrm{~h}$, followed by $1000^{\circ} \mathrm{C}$ in $\mathrm{O}_{2}$ for $2 \mathrm{~h}$, then slow cooled in $\mathrm{O}_{2}$. The resistivity versus temperature was measured in the standard four-point configuration by both ac and dc methods. The $10 \%-90 \%$ transitions are at $92-91 \mathrm{~K}$, and $94-92 \mathrm{~K}$ for samples 1 and 2, respectively. Zero resistance is reached at 90 and $91 \mathrm{~K}$, correspondingly. The x-ray diffraction pattern of sample 1 displays multiple phases. However, a substantially simpler diffraction pattern was reproduced in sample 2, suggesting both samples share a common superconducting phase. Samples for TEM were prepared by mechanical polishing followed by ion milling. TEM observations were made in a JEOL 2000FX 200-kV electron microscope equipped with both liquid-nitrogen and
liquid-He sample stages. All the electron diffraction patterns shown in this work were obtained at a temperature $\sim 110 \mathrm{~K}$.

The first sample we studied was the mixed-phase $\mathrm{Y}_{1.2} \mathrm{Ba}_{0.8} \mathrm{CuO}_{4-\delta}$. TEM examinations reveal two types of grains with very distinct appearance. Type $A$, which occupies about $70 \%$ of the sample area, is free of any obvious line or planar defects, whereas type $B$, which occupies about $30 \%$ of the area, is densely packed with somewhat regular and parallel planar defects. An electron micrograph showing the two types of grains is shown in Fig. 1. Further studies of the pure-superconducting-phase $\mathrm{YBa}_{2} \mathrm{Cu}_{3} \mathrm{O}_{9-\delta}$ system reveal that type- $B$ grains are predominant and we therefore conclude that type- $B$ grains with the presence of parallel antiphase boundaries are the superconducting phase. However, we note that the separation of the antiphase boundaries is larger $(<1000 \AA)$ in the pure-phase sample.

In Figs. 2 and 3 we show electron diffraction patterns

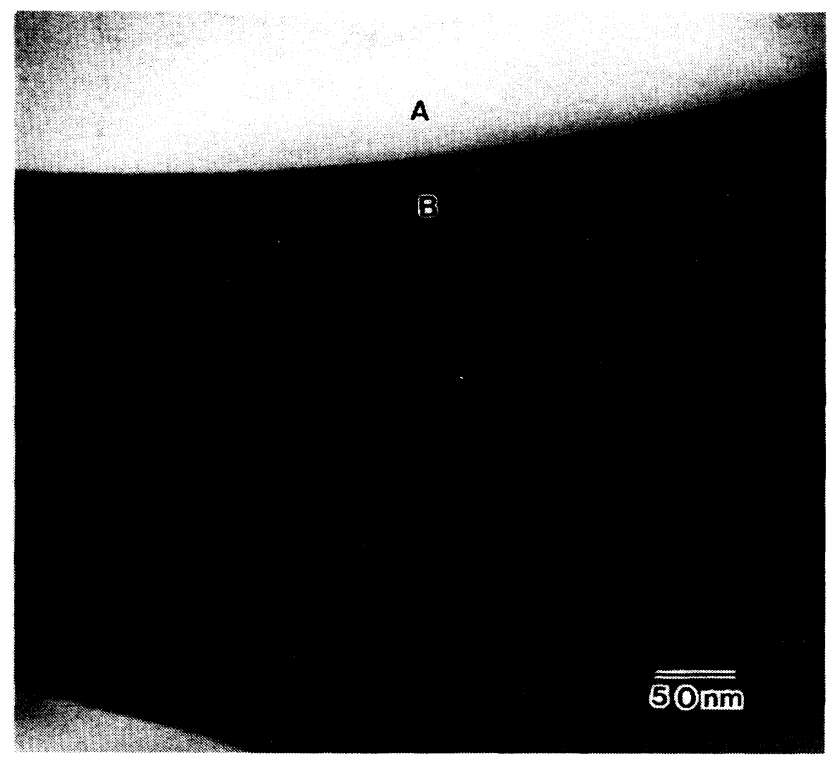

FIG. 1. Bright-field image showing two types of grains in the mixed-phase $\mathrm{Y}_{1.2} \mathrm{Ba}_{0.8} \mathrm{CuO}_{4-\delta}$ system. Grain $A$ shows no obvious line or planar defect and grain $B$, which is the superconducting phase, is packed with antiphase domain boundaries. 

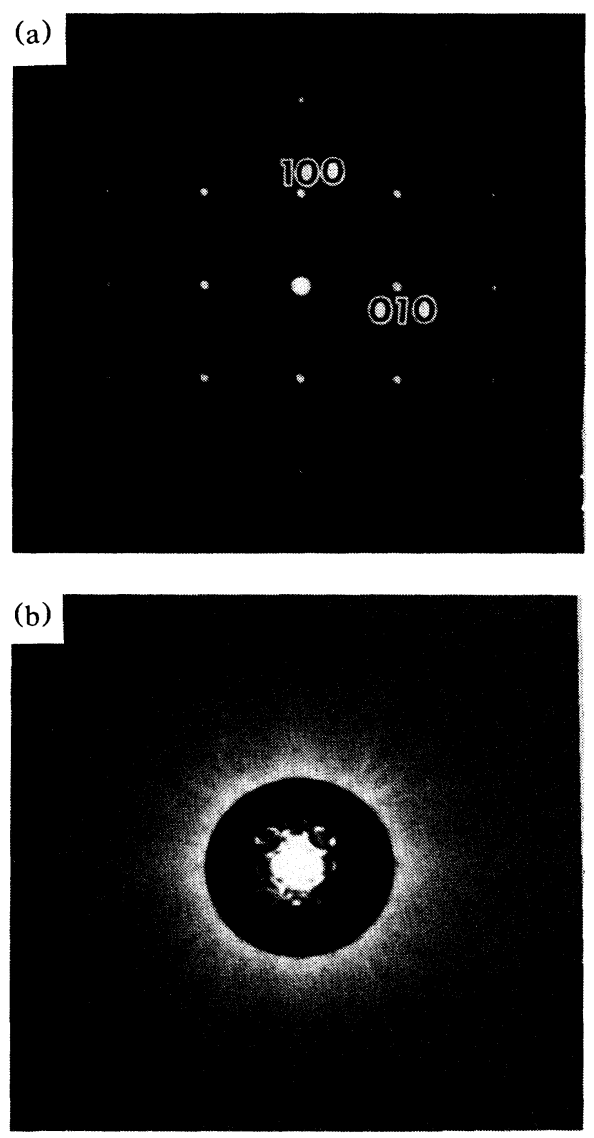

FIG. 2. [001] zone-axis electron diffraction patterns with (a) parallel-beam illumination and (b) convergent-beam illumination (CBED). Note that a much longer exposure time was used to bring out details of the diffraction disks near the central region in the CBED pattern.

obtained along two different zone axes. Two types of electron diffraction patterns are shown in Figs. 2 and 3. Figs. 2(a) and 3(a) were obtained with parallel-beam illumination, whereas Figs. 2(b) and 3(b) were obtained with convergent-beam illumination with a $\sim 200-\AA$ probe on the sample. As a result of beam convergence, diffraction disks instead of sharp diffraction spots were obtained in Figs. 2(b) and 3(b). Figure 2(a) suggests lattice plane spacings of 3.80 and $3.86 \AA$ perpendicular to the zone axis, whereas the first-order Laue zone (FOLZ) in Fig. 2(b) gives a spacing of $11.55 \AA$ in the direction along the zone axis. We note that the intensity of the FOLZ is unusually low. The reason for this is not clear at present. Figure 3 was actually obtained by tilting the sample $33.2^{\circ}$ with respect to the $3.86-\AA$ axis. We find that it is possible to index the diffraction patterns of Figs. 2(a) and $3(\mathrm{a})$ with a primitive orthorhombic cell of $a=3.80 \AA, b=3.86$ $\AA$, and $c=11.55 \AA$. The structure agrees very well with the $x$-ray results within the accuracy of the electron diffraction. We find that the zone axes for Figs. 2 and 3 are [001] and [20 $\overline{1}$ ], respectively. Convergent-beam electron diffraction (CBED) patterns such as those shown in
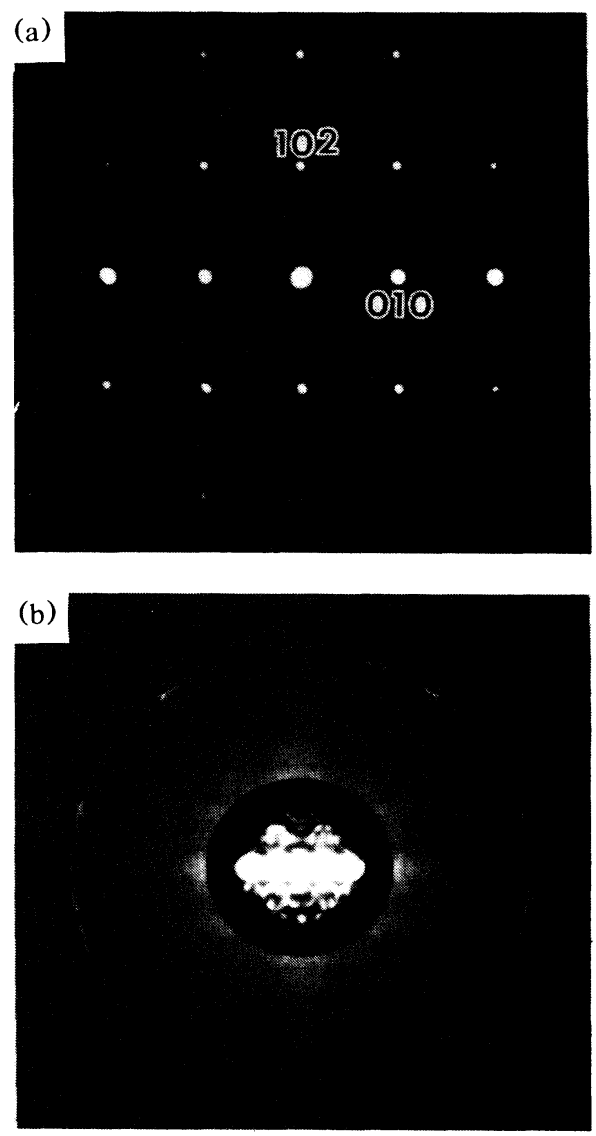

FIG. 3. [201] zone-axis electron diffraction patterns with (a) parallel beam illumination and (b) convergent-beam illumination (CBED). Note that a much longer exposure time was used to bring out details of the diffraction disks near the central region in the CBED pattern.

Figs. 2(b) and 3(b) contain the point-group symmetry information of the crystal structure. ${ }^{9}$ By close examinations of the symmetries of CBED patterns along [001] and [20ī] zone axes [Figs. 2(b) and 3(b)] we find that there exists only one mirror plane perpendicular to the $b$ axis. Following the well-established procedure for point-group determination from CBED patterns, ${ }^{9}$ we conclude that the point group of the superconducting phase is $m 2 m$. The space group is therefore $P m 2 m$.

Let us now return to the antiphase boundary structure in the superconducting grains as shown in Fig. 1. It is common in many metallic alloy systems that dense antiphase and/or twinned boundaries would form when noncubic ordered phases were precipitated from the cubic solid solutions. ${ }^{10}$ The driving force for the formation of these antiphase-twinned boundaries is the minimization of transformation strain energies. The antiphase boundaries which are parallel to the $c$ axis were also found to run parallel to the [110] direction. CBED [001] zone-axis patterns obtained from two adjacent domains [Figs. 4(a) and 4(b)] give identical patterns but with symmetry rotated $90^{\circ}$ to each other. We therefore have an antiphase 

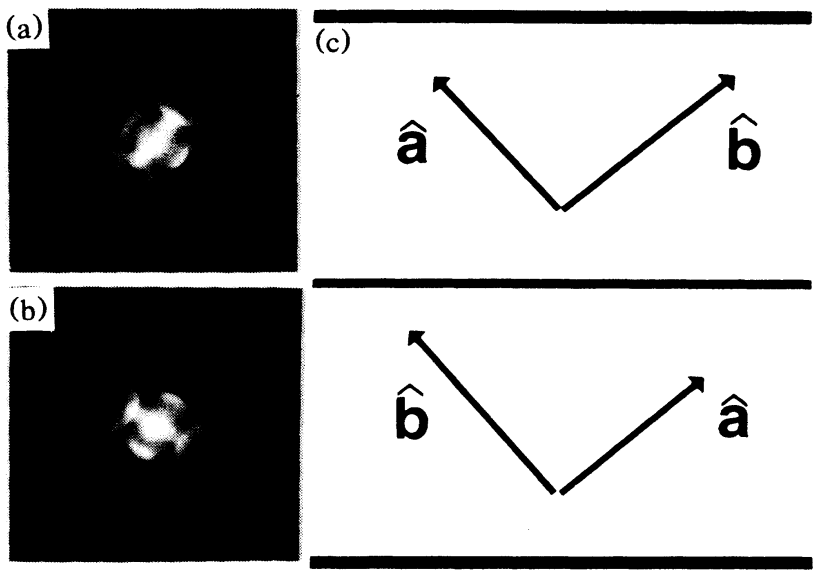

â

FIG. 4. (a) and (b) magnified CBED patterns along the [001] zone axis obtained from two adjacent domains across an antiphase boundary. Note the symmetry of CBED patterns are $90^{\circ}$ to each other. (c) Schematic showing the change of domain orientation across antiphase domain boundaries. boundary across which the orthorhombic $a-b$ axes are switched and become $b-a$ axes as indicated in Fig. 4(c). This kind of antiphase boundary structure seems to suggest that the crystal structure at some higher temperature should be tetragonal as in the case of perovskite $\mathrm{La}_{2} \mathrm{CuO}_{4}$ crystal which exhibits a tetragonal-to-orthorhombic transition at $260^{\circ} \mathrm{C}$. ${ }^{11}$ Similarly, the high- $T_{c}$ superconductor $\mathrm{La}_{1.85} \mathrm{Sr}_{0.15} \mathrm{CuO}_{4}$ has a tetragonal-to-orthorhombic phase transition for $T \lesssim 200 \mathrm{~K} .{ }^{12}$ We have observed similar antiphase boundaries in both $\mathrm{La}_{2} \mathrm{CuO}_{4}$ (Ref. 13) and $\mathrm{La}_{1.85} \mathrm{Sr}_{0.15} \mathrm{CuO}_{4}{ }^{14}$ We note that antiphase boundaries were also seen in the $\mathrm{Ba}-\mathrm{Bi}-\mathrm{Pb}-\mathrm{O}$ superconductors. ${ }^{15}$ It is possible that the formation of antiphase boundaries may be closely related to the attainment of high superconducting transition temperatures in this class of oxide superconductors.

In summary, we have observed the formation of antiphase boundaries in the superconducting phase of $\mathrm{Y}-\mathrm{Ba}$ $\mathrm{Cu}-\mathrm{O}$ system. The orthorhombic $a-b$ axes are found to alternate across a antiphase boundary. We have also determined the space group of this phase as $P m 2 m$.
${ }^{1}$ J. G. Bednorz and K. A. Müller, Z. Phys. B 64, 189 (1986).

${ }^{2}$ H. Takagi, S. Uchida, K. Kitazawa, and S. Tanaka, Jpn. J. Appl. Phys. Lett. (to be published).

${ }^{3}$ R. J. Cava, B. Batlogg, R. B. van Dover, and E. A. Rietman, Phys. Rev. Lett. 58, 408 (1987).

${ }^{4}$ C. W. Chu, P. H. Hor, R. L. Meng, L. Gas, E. J. Huang, and Y. Q. Wang, Phys. Rev. Lett. 58, 405 (1987).

${ }^{5}$ J. M. Tarascon, L. H. Greene, W. R. McKinnon, G. W. Hull, and T. H. Geballe, Science 235, 1373 (1987).

${ }^{6}$ M. K. Wu, J. R. Ashburn, C. J. Torng, P. H. Hor, R. L. Meng, L. Gao, Z. J. Huang, Y. Q. Wang, and C. W. Chu, Phys. Rev. Lett. 58, 908 (1987).

${ }^{7}$ R. J. Cava, B. Batlogg, R. B. van Dover, D. W. Murphy, S. Sunshine, T. Siegrist, J. P. Remeika, E. A. Rietman, Z. Zahurak, and G. P. Espinosa, Phys. Rev. Lett. 58, 1676 (1987).
${ }^{8}$ S. H. Liou, M. Hong, J. Kwo, and C. L. Chien (unpublished).

${ }^{9}$ For example, see, J. W. Steeds, Introduction to Analytical Electron Microscopy (Plenum, New York, 1979), Chap. 15.

${ }^{10} \mathrm{H}$. Warlimont, in Electron Microscopy and Structure of Materials, edited by G. Thomas (Univ. of California Press, Los Angeles, 1972), p. 505.

11 J. M. Longo and P. M. Raccah, J. Solid State Chem. 6, 526 (1973).

${ }^{12}$ R. J. Cava, A. Santoro, D. W. Johnson, and W. W. Rhodes (unpublished).

${ }^{13}$ C. H. Chen, D. J. Werder, and J. P. Remeika (unpublished).

${ }^{14}$ C. H. Chen, D. J. Werder, and R. J. Cava (unpublished).

${ }^{15} \mathrm{C}$. H. Chen and J. P. Remeika (unpublished). 


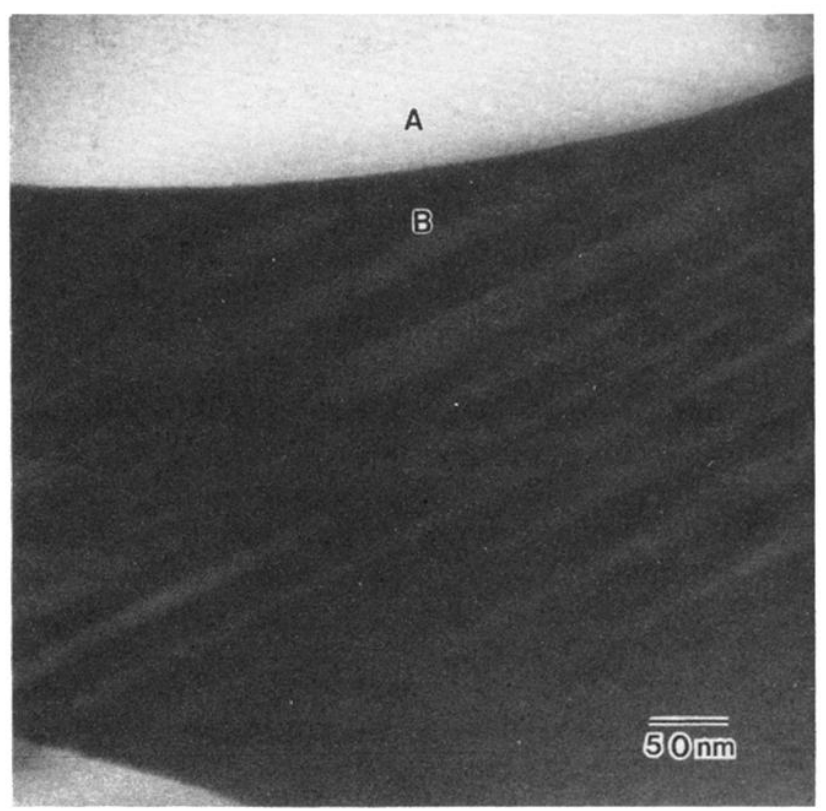

FIG. 1. Bright-field image showing two types of grains in the mixed-phase $\mathrm{Y}_{1.2} \mathrm{Ba}_{0.8} \mathrm{CuO}_{4-\delta}$ system. Grain $A$ shows no obvious line or planar defect and grain $B$, which is the superconducting phase, is packed with antiphase domain boundaries. 
(a)

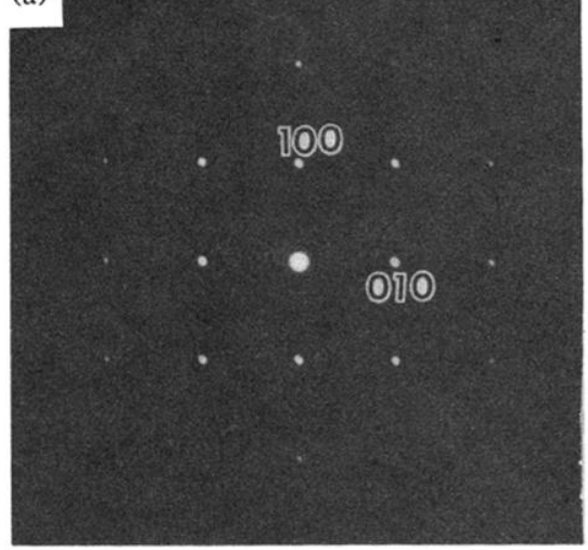

(b)

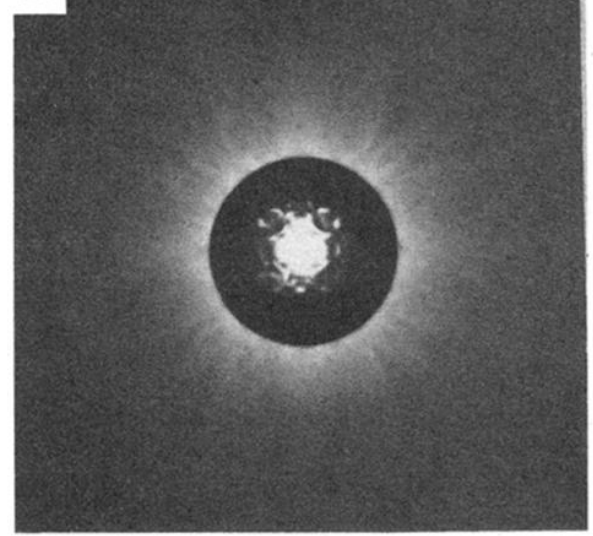

FIG. 2. [001] zone-axis electron diffraction patterns with (a) parallel-beam illumination and (b) convergent-beam illumination (CBED). Note that a much longer exposure time was used to bring out details of the diffraction disks near the central region in the CBED pattern. 

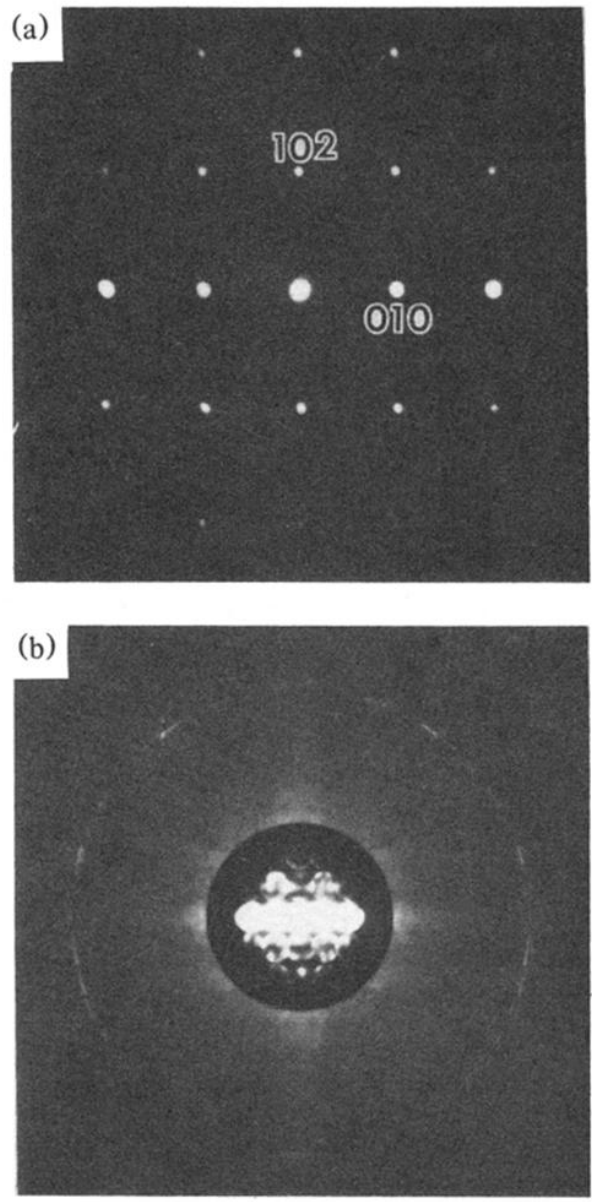

FIG. 3. [201] zone-axis electron diffraction patterns with (a) parallel beam illumination and (b) convergent-beam illumination (CBED). Note that a much longer exposure time was used to bring out details of the diffraction disks near the central region in the CBED pattern. 

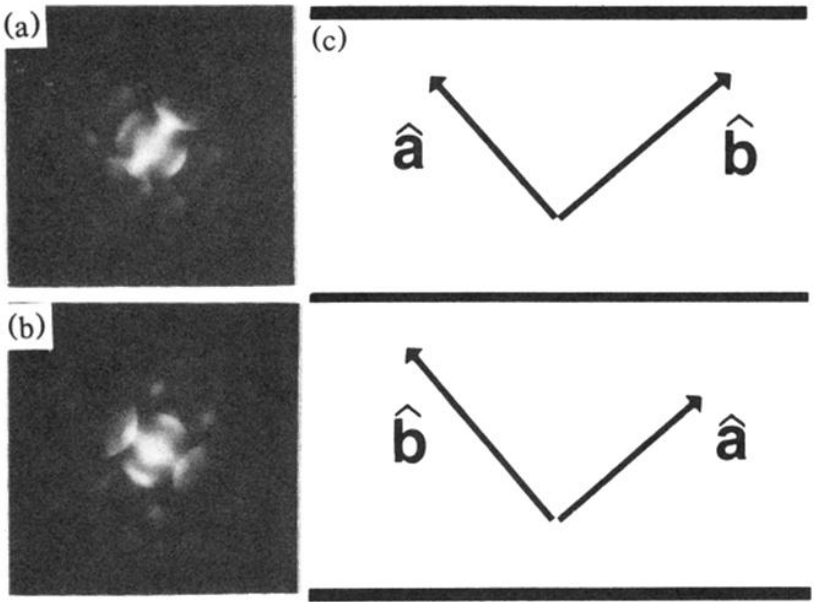

FIG. 4. (a) and (b) magnified CBED patterns along the [001] zone axis obtained from two adjacent domains across an antiphase boundary. Note the symmetry of CBED patterns are $90^{\circ}$ to each other. (c) Schematic showing the change of domain orientation across antiphase domain boundaries. 